Case Report

\title{
Mitral Valve Repair in a Patient with Acromegaly
}

\author{
Tadashi Omoto, MD, PhD, Takeo Tedoriya, MD, PhD, Masaya Oi, MD, Naoko Nagano, MD, \\ Tadamasa Miyauchi, MD, PhD, and Noboru Ishikawa, MD, PhD
}

\begin{abstract}
We report on a successful mitral valve (MV) repair and modified Cox maze procedure in a 35-year-old male patient with acromegaly, associated with severe mitral regurgitation and atrial fibrillation. He underwent a transsphenoidal adenomectomy, 7 months after the cardiac operation, and IGF-I level was normalized postoperatively. Valvular disease in patients with acromegaly is associated with hormonal activity, and control of growth hormone and insulin-like growth factor I excesses is important in the long-term durability of mitral valve repair.
\end{abstract}

Keywords: mitral valve repair, acromegaly

\section{Introduction}

Chronic elevated levels of growth hormone and insulin-like growth factor (IGF)-I secretion are known to impair cardiac morphology and performance. Indeed, left ventricular (LV) hypertrophy is extremely common in patients diagnosed with acromegaly, and interstitial fibrosis constitutes the main abnormality. ${ }^{1)}$ Coexistence of hypertension further exacerbates LV hypertrophy. Previous studies have also shown a high prevalence of cardiac valve abnormalities in patients with acromegaly, ${ }^{2,3}$ and abnormalities in matrix regulation have been implicated in the pathogenesis of aortic and mitral valve (MV) disease. ${ }^{4,5)}$ Ohtsuka and colleagues reported ring fragility and leaflet disarray in MVs, suggesting that valve replacement was more likely to achieve a better outcome than valve repair. ${ }^{6}$ In contrast, Cable and colleagues reported success in surgically repairing MVs in patients with acromegaly including active endocrinopathy.7) Here,

Division of Thoracic and Cardiovascular Surgery, Department of Surgery, Showa University, Tokyo, Japan

Received: March 30, 2011; Accepted: April 26, 2011

Corresponding author: Tadashi Omoto, MD, PhD. Division of Thoracic and Cardiovascular Surgery, Department of Surgery, Showa University, Hatanodai 1-5-8, Shinagawa-ku, Tokyo 1420064, Japan

Email: tomoto@med.showa-u.ac.jp

(C)2011 The Editorial Committee of Annals of Thoracic and Cardiovascular Surgery. All rights reserved. we report on a successful MV repair and modified Cox maze procedure in a patient with acromegaly, associated with severe mitral regurgitation and atrial fibrillation.

\section{Case Report}

A 35-year-old man was admitted to our facility for dyspnea on exertion. On admission, his weight was $75 \mathrm{~kg}$ and height was $178 \mathrm{~cm}$, and he exhibited the characteristic acromegalic appearance of frontal bossing, increased heel pad thickness $(21 \mathrm{~mm})$, mandibular enlargement and coarse facial features. No neurological symptoms or signs were noted.

On auscultation, a grade IV holosystolic murmur was heard at the apex. Chest roentgenogram showed mild pulmonary congestion and moderate cardiomegaly (cardiothoracic ratio: 56\%) and electrocardiogram showed atrial fibrillation. Echocardiography revealed LV dilatation (end-diastolic dimension /end-systolic dimension = $71 \mathrm{~mm} / 41 \mathrm{~mm})$ and left atrial dilatation $(52 \mathrm{~mm})$. LV ejection fraction was $53 \%$ (Fig. 1a). Intraventricular septum thickness and posterior wall thickness were $8 \mathrm{~mm}$ and $5 \mathrm{~mm}$, respectively. Large $\mathrm{P}_{2}$ scallop prolapse was noted, along with severe mitral regurgitation (Fig. 1b). Laboratory studies revealed normal levels of hemoglobin A1c (5.4\%) but elevated levels of brain natriuretic peptide (109 pg/ml). Plasma levels of growth hormone (GH) were within the normal range $(1.11 \mathrm{ng} / \mathrm{ml})$, but levels of IGF-I 

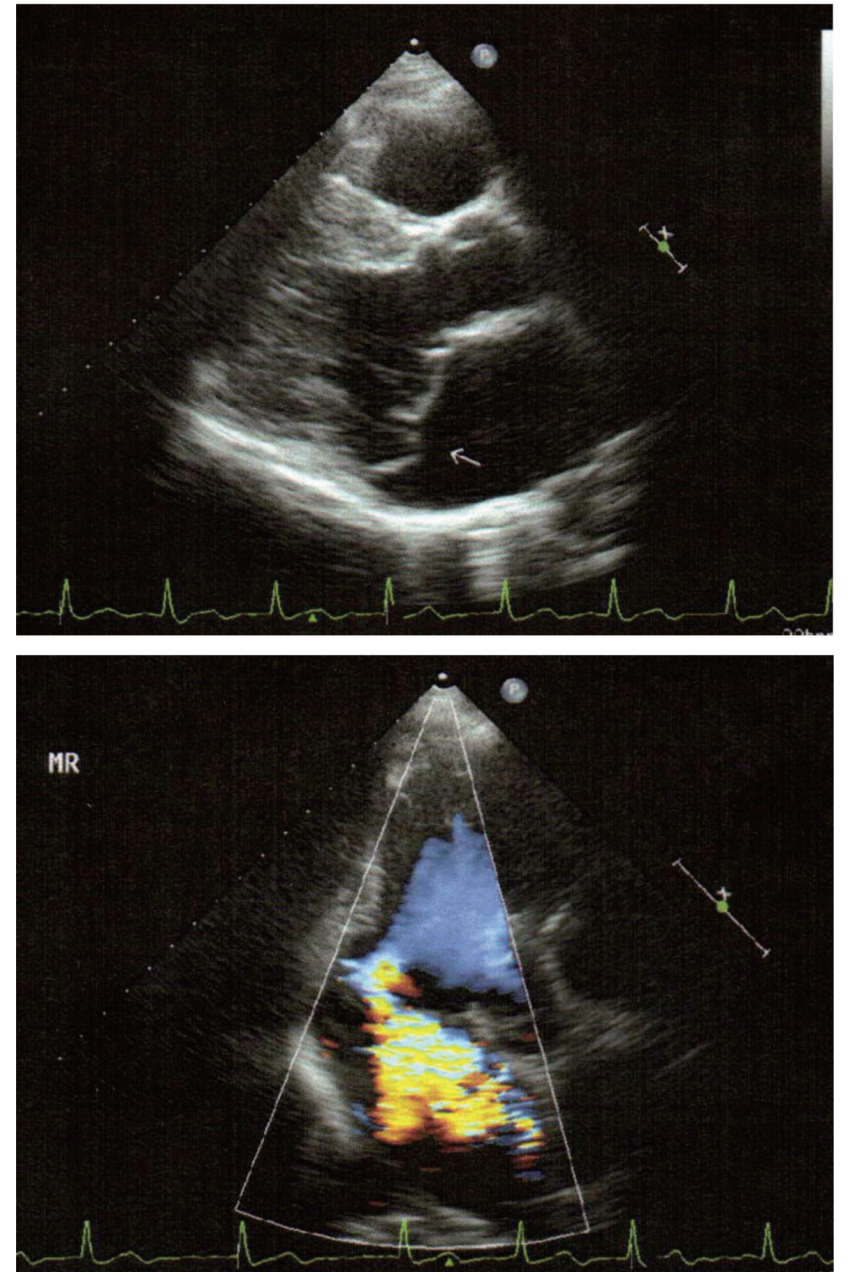

Fig. 1

a

a: Preoperative transthoracic echocardiogram. Parasternal long-axis view demonstrating prolapse of the posterior leaflet.

b: Apical view demonstrating severe mitral regurgitation.

were elevated (418 ng/ml). GH levels were not suppressed by oral glucose load ( $75 \mathrm{~g}$ ) but were suppressed after a bromocriptine suppression test. Magnetic resonance imaging of the brain revealed a pituitary microadenoma without compression of the optic nerve, and the patient was ultimately diagnosed with acromegaly associated with MV prolapse and a functional pituitary adenoma.

MV operation was planned before resection of the pituitary tumor, as endocrinological activity was low and delay of MV operation is associated with high risk in patients with acromegaly. ${ }^{6}$ Surgery was performed under standard cardiopulmonary bypass. $\mathrm{P}_{2}$ scallop prolapse without torn chordae was identified, and the MV was repaired by quadrant leaflet resection and annuloplasty

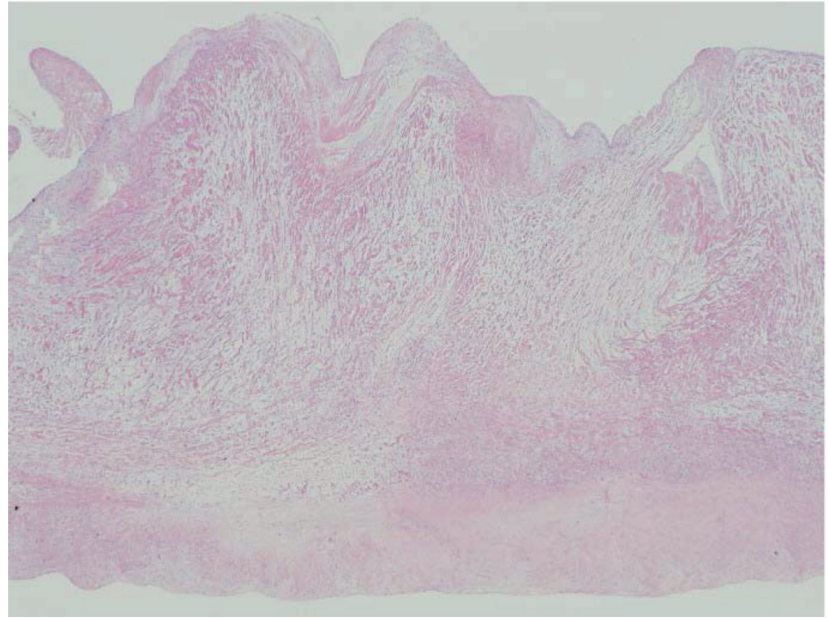

Fig. 2 Histologic section of posterior valve leaflet. The valve is thickened with marked myxomatous proliferation.

with a Carpenter-Edwards physioring (Edwards Lifesciences, Irvine, CA, USA). Tricuspid annuloplasty was performed using an Edwards MC3 (Edwards Lifesciences). A modified Cox maze procedure using radiofrequency ablation was also performed.

Intraoperative transesophageal echocardiography found no residual mitral regurgitation, and sinus rhythm was observed on electrocardiogram. Postoperative course was uneventful. Pathological examination revealed a myxomatous change in the excised mitral leaflet (Fig. 2). The patient subsequently underwent transsphenoidal adenomectomy 7 months after the cardiac operation. IGF-I levels were normalized postoperatively $(260 \mathrm{ng} / \mathrm{ml})$. Pathological examination of the pituitary tumor found findings suggestive of a $\mathrm{GH}$-secreting pituitary tumor. Twenty months after the cardiac operation, the patient showed no recurrence of mitral regurgitation, being classified as New York Heart Association functional class I and showing stable sinus rhythm.

\section{Discussion}

Prevalence of abnormalities in mitral and aortic valves is high in patients with acromegaly. Indeed, an autopsy study showed that the prevalence of valvular pathology was $19 \%$ in patients with acromegaly. ${ }^{1)}$ While several previous studies have reported on MV operation in patients with acromegaly, MV repair remains controversial. Ohtsuka and colleagues reported on five patients who underwent MV replacement and concluded that MV replacement was more effective than MV repair considering the degeneration and fragility of valvular rings. $\left.{ }^{6}\right)$ These 
authors also emphasized postoperative low output syndrome associated with LV hypertrophy. Nevertheless, Cable and colleagues successfully conducted MV repair in three patients with acromegaly. ${ }^{7)}$

Determining whether or not MV repair should be recommended in patients with acromegaly is difficult given the small patient population and uncertainty regarding the long-term durability of MV repair in these patients. Myxomatous degeneration in acromegaly resembles that found in connective tissue diseases. For example, excess leaflet tissue, chordae elongation, and annular dilatation are all characteristics found in patients with Marfan syndrome. ${ }^{8)}$ Promising long-term results have been achieved following MV repair in patients with Marfan syndrome despite the underlying disease process. ${ }^{8)}$

Endocrinologic state of the disease is an important factor in valvular disease, in patients with acromegaly. ${ }^{9)}$ Active acromegaly alters cardiac structure and performances. Prevalence of MV disease is increased in patients with active acromegaly and patients with inactive acromegaly, defined as medically well-controlled GH excess or surgically cured, exhibit relatively stable MV function. ${ }^{9)}$ Systemic hypertension in patients with active acromegaly may be closely linked to deterioration of $\mathrm{MV}$ regurgitation. In addition, persistent long-term exposure to elevated levels of GH predisposes patients to accelerated degenerative valvular changes and cardiomyopathy. MV repair in patients with inactive acromegaly may be more effective long-term treatment than replacement. However, cardiac operation was conducted before resection of the functional pituitary tumor in the present case because symptomatic, severe mitral regurgitation carries a high risk in patients with acromegaly, and, therefore, early-as-possible cardiac operation is recommended. ${ }^{6}$ Resection of the functional pituitary tumor allowed for control of GH levels and IGF-I excess, which is important in achieving long-term durability with MV repair.
In conclusion, acromegaly is not a contraindication for MV repair, provided the endocrinologic state is inactive. An operation for functional pituitary tumor should be performed early after the MV repair to prevent progression of valvular degeneration and LV hypertrophy.

\section{References}

1) Lie JT. Pathology of the heart in acromegaly: anatomic findings in 27 autopsied patients. Am Heart J 1980; 100: $41-52$.

2) Colao A, Spinelli L, Marzullo P, et al. High prevalence of cardiac valve disease in acromegaly: an observational, analytical, case-control study. J Clin Endocrinol Metab 2003; 88: 3196-201.

3) Pereira AM, van Thiel SW, Lindner JR, et al. Increased prevalence of regurgitant valvular heart disease in acromegaly. J Clin Endocrinol Metab 2004; 89: 71-5.

4) Rabkin E, Aikawa M, Stone JR, et al. Activated interstitial myofibroblasts express catabolic enzymes and mediate matrix remodeling in myxomatous heart valves. Circulation 2001; 104: 2525-32.

5) Jaffe AS, Geltman EM, Rodey GE, et al. Mitral valve prolapse: a consistent manifestation of type IV EhlersDanlos syndrome. The pathogenetic role of the abnormal production of type III collagen. Circulation 1981; 64: $121-5$.

6) Ohtsuka G, Aomi S, Koyanagi H, et al. Heart valve operation in acromegaly. Ann Thorac Surg 1997; 64: 390-3.

7) Cable DG, Dearani JA, O'Brien T, et al. Surgical treatment of valvular heart disease in patients with acromegaly. J Heart Valve Dis 2000; 9: 828-31.

8) Fuzellier JF, Chauvaud SM, Fornes P, et al. Surgical management of mitral regurgitation associated with Marfan's syndrome. Ann Thorac Surg 1998; 66: 68-72.

9) van der Klaauw AA, Bax JJ, Roelfsema F, et al. Uncontrolled acromegaly is associated with progressive mitral valvular regurgitation. Growth Hormon \& IGF Research 2006; 16: 101-7. 